Proyecciones Journal of Mathematics

Vol. 30, No 3, pp. 303-318, December 2011.

Universidad Católica del Norte

Antofagasta - Chile

DOI: 10.4067/S0716-09172011000300003

\title{
On certain isotopic maps of central loops
}

\author{
JOHN OLUSOLA ADENIRAN \\ UNIVERSITY OF AGRICULTURE, NIGERIA \\ YACUB TUNDE OYEBO \\ LAGOS STATE UNIVERSITY, NIGERIA \\ and \\ DAABO MOHAMMED \\ UNIVERSITY FOR DEVELOPMENT STUDIES, GHANA \\ Received : October 2010. Accepted : September 2011
}

\begin{abstract}
It is shown that the Holomorph of a C-loop is a C-loop if each element of the automorphism group of the loops is left nuclear. Condition under which an element of the Bryant-Schneider group of a C-loop will form an automorphism is established. It is proved that elements of the Bryant-Schneider group of a C-loop can be expressed a product of pseudo-automorphisms and right translations of elements of the nucleus of the loop. The Bryant-Schneider group of a $C$-loop is also shown to be a kind of generalized holomorph of the loop.
\end{abstract}

2000 Mathematics Subject Classification : Primary 20NO5; Secondary 08405.

Keywords : Central loop, isotopism, autotopism, Bryant-Schneider group. 


\section{Introduction}

Central loops(C-loops) are loops which satisfy one of the identities called "Central identity" as named by F. Fenyves [9], [10]. Closely related to the central identity are left central(LC) and right central (RC) identities. The expressions for the mentioned identities are as follows;

$$
\begin{gathered}
(y x \cdot x) z=y(x \cdot x z) \text { central identity } \\
i . x x \cdot y z=(x \cdot x y) z \equiv \\
i i .(x \cdot x y) z=x(x \cdot y z) \equiv \\
i i i .(x x \cdot y) z=x(x \cdot y z) \\
\text { LC- identities } \\
i . y z \cdot x x=y(z x \cdot x) \equiv \\
i i .(y z \cdot x) x=y(z x \cdot x) \equiv \\
i i i .(y z \cdot x) x=y(z \cdot x x) \\
\text { RC- identities }
\end{gathered}
$$

Recently Phillips and Vojtechovsky [20], found out that in addition to the identities above, LC and RC identity can also be defined respectively by,

$$
(y \cdot x x) z=y(x \cdot x z) \text { and }(y x \cdot x) z=y(x x \cdot z)
$$

C-loops are one of the least studied loops. Few publications that have considered C-loops include Fenyves [9], [10], Phillips and Vojtechovsky [18] [20] [19], Chein [5]. The difficulty in studying them is as a result of the nature of their identities when compared with other Bol-Moufang identities(the element occurring twice on both sides has no other element separating it from itself).

\section{Preliminaries}

Theorem 2.1. ([10], [20]) Let $(L, \cdot)$ be an LC-loop(RC-loop). Then:

1. $(L, \cdot)$ is a left (right) alternative loop,

2. $(L, \cdot)$ is a left (right) inverse property loop,

3. $(L, \cdot)$ is a left (right) nuclear square loop,

4. $(L, \cdot)$ is a left (right) power alternative loop, 
5. $(L, \cdot)$ is a middle square loop,

6. $(L, \cdot)$ is power associative loop.

Definition 2.1. A triple $(\alpha, \beta, \gamma)$ of bijections is called an isotopism of loop $(L, \cdot)$ onto a loop $(H, \circ)$ provided $x \alpha \circ y \beta=(x \cdot y) \gamma \forall x, y \in L$. $(H, \circ)$ is called an isotope of $(L, \cdot)$. The loops $(L, \cdot)$ and $(H, \circ)$ are said to be isotopic to each other.

Definition 2.2. Let $\alpha$ and $\beta$ be a permutation of $L$ and let $\iota$ denotes the identity map on $L$. Then $(\alpha, \beta, \iota)$ is a principal isotopism of a loop $(L, \cdot)$ onto a loop $(L, \circ)$ which imply that $(\alpha, \beta, \iota)$ is an isotopism of $(L, \cdot)$ onto $(L, \circ)$.

Definition 2.3. An isotopism of $(L, \cdot)$ onto $(L, \cdot)$ is called an autotopism of $(L, \cdot)$. The group of autotopisms of $L$ is denoted by $A(L)$.

Remark 2.1. The components of isotopism are usually denoted by lower case Greek letters. However, we shall denote the components of autotopism by capital letters, thus if $T=(U, V, W)$ is an autotopism of a loop $(L, \cdot)$, then

$$
x U \cdot y V=(x y) W, \forall x, y \in L .
$$

The set of all autotopism of a loop is a group with the inverse of $T T^{-1}=$ $(U, V, W)^{-1}=\left(U^{-1}, V^{-1}, W^{-1}\right)$. The identity element of the group being $(I, I, I)$ where $I$ is the identity map of $L$. If $T=(U, U, U)$, then $T$ is called the automorphism $(L, \cdot)$

Definition 2.4. If $\langle U, V, W\rangle$ is autotopism of an inverse property loop $(L,$.$) then \langle W, J V J, U\rangle$ and $\langle J U J, W, V\rangle$ are autotopism of $L$. Moreover if $\langle U, V, W\rangle=\left\langle S, S R_{c}, S R_{c}\right\rangle$ the $S$ is called a pseudoautomorphism of $L$ with companion $c$. The set of all pseudoautomorphisms of $L$ is denoted by $P S(L,$.$) .$

Definition 2.5. Let $(L, \cdot)$ be an inverse property loop with the nucleus denoted by $N$. Then an automorphism $\alpha$ of $(L, \cdot)$ is left nuclear if $f a \alpha \cdot a^{-1} \in$ $N$ for all $a \in L$.

Definition 2.6. Let $(L,$.$) be a loop and B S(L,$.$) be the set of all permu-$ tations $\theta$ of $Q$ such that

$$
<\theta R_{g}^{-1}, \theta L_{f}^{-1}, \theta>
$$

is an autotopism of $(L,$.$) for some f, g \in L$, then $B S(L,$.$) is called the$ Bryant-Schneider group of the loop. 
Definition 2.7. Let $(L, \cdot)$ be a loop, $A(L)$ a group of automorphisms of loop $(L, \cdot)$ and let $H H=A(L) \times L$ and define

$$
(\alpha, x) o(\beta, y)=(\alpha \beta, x \beta \cdot y)
$$

$\forall(\alpha, x),(\beta, y) \in H$. Then the loop $(H, o)$ is called the $A(L)$-holomorph of $(L, \cdot)$ or simply holomorphy of $(L,$.$) .$

\section{Holomorphy}

Theorem 3.1. Let $(L, \cdot)$ be a an $L C$-loop and $A(L)$ be a group of automorphism of $(L, \cdot)$. Then the $A(L)$-holomorph $(H, o)$ of $(L, \cdot)$ is an LC-loop if and only if

$$
(x \alpha \cdot x y) z=x \alpha(x \cdot y z)
$$

$\forall x, y, z \in L$ and $\forall \alpha \in A(L)$.

\section{Proof.}

Suppose $\mathrm{A}(\mathrm{L})$-holomorph $(\mathrm{H}, \mathrm{o})$ of $(L, \cdot)$ is an LC-loop we have

$$
\{(\alpha, x) o[(\alpha, x) o(\beta, y)]\} o(\gamma, z)=(\alpha, x) o\{(\alpha, x) o[(\beta, y) o(\gamma, z)]\}
$$

$\forall x, y, z \in L$ and $\forall \alpha, \beta, \gamma \in A(L)$. Thus

$$
\begin{gathered}
\{(\alpha, x) o(\alpha \beta, x \beta \cdot y)\} o(\gamma, z)=(\alpha, x) o\{(\alpha, x) o(\beta \gamma, y \gamma \cdot z)\} \\
\{\alpha \cdot \alpha \beta, x \alpha \beta \cdot(x \beta \cdot y)\} o(\gamma, z)=(\alpha, x) o\{(\alpha \cdot \beta \gamma, x \beta \gamma \cdot(y \gamma \cdot z))\} \\
\{(\alpha \cdot \alpha \beta) \gamma,[x \alpha \beta \cdot(x \beta .
\end{gathered}
$$

$\mathrm{y})] \gamma \cdot z\}=\{\alpha(\alpha \cdot \beta \gamma), x \alpha \cdot \beta \gamma \cdot x \beta \gamma(y \gamma \cdot z)\} \forall x, y, z \in L$ and $\forall \alpha, \beta, \gamma \in$ $A(L)$. Therefore

$$
\{x \alpha \beta \cdot(x \beta \cdot y)\} \gamma \cdot z=x \alpha \cdot \beta \gamma \cdot x \beta \gamma(y \gamma \cdot z)
$$

$\forall x, y, z \in L$ and $\forall \alpha, \beta, \gamma \in A(L)$.

Therefore,

$$
\{x \alpha \cdot \beta \gamma \cdot(x \beta \gamma \cdot y \gamma)\} \cdot z=x \alpha \cdot \beta \gamma \cdot x \beta \gamma \cdot(y \gamma \cdot z)
$$

putting $\phi=\beta \gamma$, gives

$$
\{x \alpha \phi \cdot(x \phi \cdot y \gamma)\} z=x \alpha \phi \cdot x \phi(y \gamma \cdot z)
$$


hence

$$
\left\{x \alpha \cdot\left(x \cdot y \gamma \phi^{-1}\right)\right\} \cdot z \phi^{-1}=\left\{x \alpha \cdot x\left(y \gamma \phi^{-1} \cdot z \phi^{-1}\right\}\right.
$$

$\forall x, y, z \in L$ and $\forall \alpha, \phi, \gamma \in A(L)$. If we put $\bar{y}=y \gamma \phi^{-1}$ and $\bar{z}=z \phi^{-1}$, we obtain

$$
(x \alpha \cdot x \bar{y}) \bar{z}=x \alpha \cdot(x \cdot \bar{y} \bar{z})
$$

And replacing $\bar{y}$ and $\bar{z}$ by $y$ and $z$ respectively we have

$$
(x \alpha \cdot x y) z=x \alpha(x \cdot y z)
$$

$\forall x, y, z \in L$ and $\forall \alpha \in A(L)$, which is equation (3.1).

The converse is obtained by reversing the process.

Corollary 3.1. Let $(L, \cdot)$ be a loop, and $A(L)$ be the group of all automorphism of $L$, then $L$ is an LC-loop if

$$
B=\left\langle L_{x} L_{x \alpha}, I, L_{x} L_{x \alpha}\right\rangle
$$

is an autotopism of $L, \forall x, y, z \in L$ and $\forall \alpha \in A(L)$

Proof. This is a consequence of (3.1)

Theorem 3.2. Let $(L, \cdot)$ be a loop and $A(L)$ be a group of automorphism of $(L, \cdot)$. Then the $A(L)$-holomorph $(H, o)$ of $(L, \cdot)$ is an $R C$-loop if and only if

$$
y((z \cdot x \alpha) x)=(y z \cdot x \alpha) x
$$

$\forall x, y, z \in L$ and $\forall \alpha \in A(L)$.

\section{Proof.}

The procedure for the proof is like that of Theorem 3.1 above hence it is omitted.

Corollary 3.2. Let $(L, \cdot)$ be any loop and $A(L)$ be the group of all automorphisms of $L$, then $L$ is an $R C$-loop if and only if

$$
B=\left\langle I, R_{x \alpha} R_{x}, R_{x \alpha} R_{x}\right\rangle
$$

is an autotopism of $L$, for all $x, y, z \in L$ and all $\alpha \in A(L)$ 


\section{Proof.}

From 3.4)

$$
\begin{aligned}
& y((z \cdot x \alpha) x)=(y z \cdot x \alpha) x \\
& \Rightarrow y \cdot z R_{x \alpha} R_{x}=y z R_{x \alpha} R_{x}
\end{aligned}
$$

$\forall x, y, z \in L$ and $\forall \alpha \in A(L)$.

$$
\Rightarrow\left\langle I, R_{x \alpha} R_{x}, R_{x \alpha} R_{x}\right\rangle
$$

is an autotopism of $(L, \cdot) \forall x \in L$ and $\forall \alpha \in A(L)$.

Conversely, suppose (3.5) hold, then $\forall y, z \in L$ we have

$$
\begin{aligned}
& y I \cdot z R_{x \alpha} R_{x}=y z R_{x \alpha} R_{x} \\
& y((z \cdot x \alpha) x)=y z(x \alpha \cdot x)
\end{aligned}
$$

$\forall x, y, z \in L$ and $\forall \alpha \in A(L)$.

Theorem 3.3. Let $(L, \cdot)$ be a loop and $A(L)$ be a group of automorphism of $(L, \cdot)$. Then the $A(L)$-holomorph $(H, o)$ of $(L,$.$) is a C$-loop if and only if

$$
(y \cdot x \alpha) x \cdot z=y(x \alpha \cdot x z)
$$

$\forall x, y, z \in L$ and $\forall \alpha \in A(L)$.

\section{Proof.}

The procedure for the proof is like that of theorem 3.1 hence it is omitted.

Corollary 3.3. Let $(L,$.$) be a loop and A(L)$ be the group of all automorphisms of $L$, then $L$ is a $C$-loop if and only if

$$
B=\left\langle R_{x \alpha} R_{x}, L_{x \alpha}^{-1} L_{x^{-1}}, I\right\rangle
$$

is an autotopism of $L$, for all $x, y, z \in L$ and all $\alpha \in A(L)$

Proof. From (3.6)

$$
\begin{gathered}
(y \cdot x \alpha) x \cdot z=y(x \alpha \cdot x z) \\
\Rightarrow y R_{x \alpha} R_{x} \cdot z=y \cdot z L_{x} L_{x \alpha}
\end{gathered}
$$

$\forall x, y, z \in L$ and $\forall \alpha \in A(L)$.

substituting $\bar{z}=z L_{x} L_{x \alpha}$ we have

$$
y R_{x \alpha} R_{x} \cdot \bar{z} L_{(x \alpha)^{-1}} L_{x^{-1}}=y \bar{z}
$$


$\forall x, y, \bar{z} \in L$ and $\forall \alpha \in A(L)$.

$$
\Rightarrow\left\langle R_{x \alpha} R_{x}, L_{(x \alpha)^{-1}} L_{x^{-1}}, I\right\rangle
$$

is an autotopism of $(L, \cdot) \forall x \in L$ and $\forall \alpha \in A(L)$.

Conversely, suppose equation (3.7) is an autotopism of $(L, \cdot)$, therefore $\forall y, z \in L$ we have

$$
\begin{gathered}
y R_{x \alpha} R_{x} \cdot z L_{x \alpha}^{-1} L_{x^{-1}}=y z \cdot I \\
y R_{x \alpha} R_{x} \cdot \bar{z}=y \cdot \bar{z} L_{x} L_{x \alpha} I \\
(y \cdot x \alpha) \bar{z}=y(x \alpha \cdot x \bar{z})
\end{gathered}
$$

$\forall x, y, \bar{z} \in L$ and $\forall \alpha \in A(L)$ hence $(L, \cdot)$ is a C-loop.

\subsection{Nuclear Automorphism}

Theorem 3.4. Let $(L, \cdot)$ be a loop and $A(L)$ be a group of automorphism of $(L, \cdot)$. Then the $A(L)$-holomorph $(H, o)$ of $(L, \cdot)$ is a C-loop iff $(L, \cdot)$ is a $C$-loop and each $\alpha \in A(L)$ is a left nuclear automorphism of $(L, \cdot)$.

Proof. Suppose $(H, o)$ is a C-loop. Since $(L, \cdot)$ is isomorphic to a subloop of $(H, o)$, it follows that $(L, \cdot)$ must be a C-loop. From Theorem (3.1), equation (3.1) holds $\forall x, y, z \in L$ and $\forall \alpha \in A(L)$. Furthermore, by Theorem (3.1) and Corollary (3.3),

$$
A(x)=\left\langle R_{x}^{2}, L_{x}^{-2}, I\right\rangle \text { and } B(x)=\left\langle R_{x} R_{x \alpha}, L_{x}^{-1} L_{x \alpha}^{-1}, I\right\rangle
$$

are autotopisms of $(L, \cdot), \forall x \in L$ and $\forall \alpha \in A(L)$. Therefore by Theorem (3.1)and we have

$A_{\lambda}(x)=\left\langle L_{x}^{-2}, I, L_{x}^{-2}\right\rangle, A_{\mu}^{-1}(x)=\left\langle I, R_{x}^{2}, R_{x}^{2}\right\rangle$, $B_{\lambda}^{-1}(x)=\left\langle L_{x \alpha} L_{x}, I, L_{x \alpha} L_{x}\right\rangle$ and $B_{\mu}(x)=\left\langle I, R_{x} R_{x \alpha}, R_{x} R_{x \alpha}\right\rangle$

are also autotopisms of $(L, \cdot), \forall x \in L$ and $\forall \alpha \in A(L)$. If these are combined we have

$$
\begin{gathered}
A_{\lambda}(x) B_{\lambda}^{-1}(x)=\left\langle L_{x}^{-2}, I, L_{x}^{-2}\right\rangle\left\langle L_{x} L_{x \alpha}, I, L_{x} L_{x \alpha}\right\rangle \\
A_{\lambda}(x) B_{\lambda}^{-1}(x)=\left\langle L_{x}^{-1} L_{x \alpha}, I, L_{x}^{-1} L_{x \alpha}\right\rangle
\end{gathered}
$$

and

$$
B_{\mu}(x) A_{\mu}^{-1}(x)=\left\langle I, R_{x \alpha} R_{x}, R_{x \alpha} R_{x}\right\rangle\left\langle I, R_{x}^{-2}, R_{x}^{-2}\right\rangle
$$




$$
\left.B_{\mu}(x) A_{\mu}^{-1}(x)=\left\langle I, R_{x \alpha} R_{x}^{-1}, R_{x \alpha}\right) R_{x}^{-1}\right\rangle
$$

as autotopisms of $(L, \cdot), \forall x \in L$ and $\forall \alpha \in A(L)$. Now if we apply (3.8) and (3.9) to $1 \cdot b$ and $a \cdot 1$ respectively, we have

$$
\begin{gathered}
1 L_{x}^{-1} L_{x \alpha} \cdot b=(1 \cdot b) L_{x}^{-1} L_{x \alpha} \\
\left(x \alpha \cdot x^{-1}\right) b=b L(x)^{-1} L_{x \alpha} \\
b L_{x \alpha} L_{x}^{-1}=b L_{x}^{-1} L_{x \alpha}
\end{gathered}
$$

and

$$
\begin{gathered}
a \cdot 1 R_{x \alpha} R_{x}^{-1}=(a \cdot 1) R_{x \alpha} R_{x}^{-1} \\
a\left(x \alpha \cdot x^{-1}\right)=a R_{x \alpha} R_{x}^{-1} \\
a R_{x \alpha \cdot x^{-1}}=a R_{x \alpha} R_{x}^{-1}
\end{gathered}
$$

and respectively we have

$$
\begin{aligned}
& L_{x \alpha \cdot x^{-1}}=L_{x}^{-1} L_{x \alpha} \\
& R_{x \alpha \cdot x^{-1}}=R_{x \alpha} R_{x}^{-1}
\end{aligned}
$$

$\forall x \in L$ and $\forall \alpha \in A(L)$. If we put equations(3.10) and (3.11) into equations(3.8) and (3.9) respectively, we have

$$
A_{\lambda}(x) B_{\lambda}^{-1}(x)=\left\langle L_{x \alpha \cdot x^{-1}}, I, L_{x \alpha \cdot x^{-1}}\right\rangle
$$

and

$$
B_{\mu}(x) A_{\mu}^{-1}(x)=\left\langle I, R_{x \alpha \cdot x^{-1}}, R_{x \alpha \cdot x^{-1}}\right\rangle
$$

$\forall x \in L$ and $\forall \alpha \in A(L)$. These therefore imply that $x \alpha \cdot x^{-1} \in N_{\lambda}(L)$ and $x \alpha \cdot x^{-1} \in N_{\rho}(L)$. Consequently, $x \alpha \cdot x^{-1} \in N(L)$ since $(L, \cdot)$ is an inverse property loop. Hence $\alpha \in A(L)$, is left nuclear.

Conversely, suppose $(L, \cdot)$ is a C-loop and each $\alpha \in A(L)$ is left nuclear. Then for each $\alpha \in A(L)$ and each $x \in L$ the element $x \alpha \cdot x^{-1} \in N_{\mu}(L)$, thus

$$
\begin{gathered}
x \alpha \cdot y=\left(\left(x \alpha \cdot x^{-1}\right) x\right) y \\
x \alpha \cdot y=\left(x \alpha \cdot x^{-1}\right) x y
\end{gathered}
$$

$\forall y \in L$

$$
y L_{x \alpha}=y L_{x} L_{x \alpha \cdot x^{-1}} \Rightarrow L_{x}^{-1} L_{x \alpha}=L_{x \alpha \cdot x^{-1}}
$$


$\forall x \in L$ and $\forall \alpha \in A(L)$. But for $\forall x \in L$ and $\forall \alpha \in A(L)$, we know that $x \alpha \cdot x^{-1} \in N_{\lambda}(L)$. Hence,

$$
C=\left\langle L_{\alpha \cdot x^{-1}}, I, L_{x \alpha \cdot x^{-1}}\right\rangle=\left\langle L_{x}^{-1} L_{x \alpha}, I, L_{x}^{-1} L_{x \alpha}\right\rangle
$$

is an autotopism of $(L, \cdot), \forall x \in L$ and $\forall \alpha \in A(L)$. But again , $A=$ $\left\langle L_{x}^{2}, I, L_{x}^{2}\right\rangle$ is an autotopism of $(L, \cdot), \forall x \in L$. Therefore,

$$
A C=\left\langle L_{x} L_{x \alpha}, I, L_{x} L_{x \alpha}\right\rangle
$$

is an autotopism of $(L, \cdot), \forall x \in L$ and $\forall \alpha \in A(L)$. So also is $(A C)_{\lambda}^{-1}=$ $\left\langle R_{x \alpha} R_{x}, L_{x \alpha}^{-1} L_{x}^{-1}, I\right\rangle$. Therefore of $y z, \forall y, z \in L$, we have

$$
y R_{x \alpha} R_{x} \cdot z L_{x \alpha}^{-1} L_{x}^{-1}=y z
$$

if we put $\bar{z}=z L_{x \alpha}^{-1} L_{x}^{-1}$, in this we have

$$
\begin{aligned}
& y R_{x \alpha} R_{x} \cdot \bar{z}=y \cdot \bar{z} L_{x} L_{x \alpha} \\
& ((y \cdot x \alpha) x) \bar{z}=y(x \alpha \cdot x \bar{z})
\end{aligned}
$$

$\forall x, y, \bar{z} \in L$ and $\forall \alpha \in A(L)$. Replacing $\bar{z}$ by z, $\forall x, y, z \in L$ and $\forall \alpha \in A(L)$ and we have a central identity. Hence, $(H, o)$ is a C-loop.

Theorem 3.5. The set $S(L)$ of all left nuclear automorphism of an C-loop $(L, \cdot)$, is a normal subgroup of the automorphism group of $(L, \cdot)$.

Proof. $S(L) \neq \emptyset$, from the Theorem 3.4 it was shown that

$$
L_{u \alpha \cdot u^{-1}}=L_{u}^{-1} L_{u \alpha}
$$

$\forall u \in L$ and $\forall \alpha \in S(L)$ (since for an inverse property loop $L, L_{u^{-1}}=L_{u}^{-1}$ $\forall u \in L)$. Then $u \alpha \cdot u^{-1} \in N_{\lambda}(L, \cdot), \forall u \in L$ and $\forall \alpha \in S(L)$. It follows then that

$$
A(\alpha, u)=\left\langle L_{u \alpha \cdot u^{-1}}, I, L_{u \alpha \cdot u^{-1}}\right\rangle=\left\langle L_{u}^{-1} L_{u \alpha}, I, L_{u}^{-1} L_{u \alpha}\right\rangle
$$

$\forall u \in L$ and forall $\alpha \in L$. Hence if $\alpha, \beta \in S(L)$, we have

$$
\begin{gathered}
A(\alpha, u) A(\beta, u \alpha)=\left\langle L_{u}^{-1} L_{u \alpha}, I, L_{u}^{-1} L_{u \alpha}\right\rangle\left\langle L_{u \alpha}^{-1} L_{u \alpha \beta}, I, L_{u \alpha}^{-1} L_{u \alpha \beta}\right\rangle \\
A(\alpha, u) A(\beta, u \alpha)=\left\langle L_{u}^{-1} L_{u \alpha \beta}, I, L_{u}^{-1} L_{u \alpha \beta}\right\rangle
\end{gathered}
$$


is an autotopism of $(L, \cdot), \forall u \in L$. Therefore $\forall y \in L$ we have

$$
\begin{gathered}
1 L_{u}^{-1} L_{u \alpha \beta} \cdot y=(1 \cdot y) L_{u}^{-1} L_{u \alpha \beta} \\
\left(u \alpha \beta \cdot u^{-1}\right) \cdot y=y L_{u}^{-1} L_{u \alpha \beta} \\
y L_{u \alpha \beta \cdot u^{-1}}=y L_{u}^{-1} L_{u \alpha \beta} \\
\Rightarrow L_{u \alpha \beta \cdot u^{-1}}=L_{u^{-1} L_{u \alpha \beta}}
\end{gathered}
$$

Thus, (3.13) into (3.12) gives

$$
A(\alpha, u) A(\beta, u \alpha)=\left\langle L_{u \alpha \beta . u^{-1}}, I, L_{u \alpha \beta . u^{-1}}\right\rangle
$$

From equation (3.14), $u \alpha \beta . u^{-1} \in N_{\lambda}(L, \cdot), \forall u \in L$, hence $u \alpha \beta . u^{-1} \in N$, for all $u \in L$ and so $\alpha \beta \in S(L)$, since $(L, \cdot)$ is an inverse property loop.

If $\alpha \in S(L)$, then $A(\alpha, u)$ is an autotopism of $(L, \cdot) \forall u \in L$, so also is $A\left(\alpha, u \alpha^{-1}\right)^{-1} \forall u \in L$, i.e

$$
\begin{gathered}
A\left(\alpha, u \alpha^{-1}\right)^{-1}=\left\langle L_{u \alpha^{-1}}^{-1} L_{u \alpha^{-1} \cdot \alpha}, I, L_{u \alpha^{-1}}^{-1} L_{\alpha^{-1} . \alpha}\right\rangle^{-1} \\
=\left\langle L_{u \alpha^{-1}}^{-1} L_{u}, I, L_{u \alpha^{-1}}^{-1} L_{u}\right\rangle^{-1} \\
=\left\langle L_{u}^{-1} L_{u \alpha^{-1}}, I, L_{u}^{-1} L_{u \alpha}^{-1}\right\rangle \\
=\left\langle L\left(u \alpha^{-1} \cdot u^{-1}\right), I, L\left(u \alpha^{-1} \cdot u^{-1}\right)\right\rangle
\end{gathered}
$$

Hence it follows that $\alpha^{-1} \in S(L)$. Thus $S(L)$ is a subgroup of the automorphism group of $(L, \cdot)$.

Let $\alpha \in S(L)$, then $u \alpha \cdot \alpha^{-1} \in N_{\lambda}(L, \cdot), \forall u \in L$ and

$$
\left(u \alpha \cdot u^{-1}\right) x y=\left(u \alpha \cdot u^{-1}\right) x . y
$$

$\forall u, x, y \in L$, if $\gamma$ is an automorphism of $(L, \cdot)$, then we have

$$
\left.\left\{u \alpha \gamma \cdot(u \gamma)^{-1}\right)\right\}(x \gamma \cdot y \gamma)=\left\{u \alpha \gamma \cdot(u \gamma)^{-1}\right\} x \gamma \cdot y \gamma
$$

$\forall u, x, y \in L$, and if we replace $\mathrm{u}$ by $u \gamma^{-1}$ in the last expression, we have

$$
\left(u \gamma^{-1} \alpha \gamma \cdot u^{-1}\right)(x \gamma \cdot y \gamma)=\left(u \gamma^{-1} \alpha \gamma \cdot u^{-1}\right) x \gamma \cdot y \gamma
$$

Thus, $u \gamma^{-1} \alpha \gamma \cdot u^{-1} \in N_{\lambda}(L, \cdot)$ and since $L$ is an inverse property loop, the three nuclei coincide, then $u \gamma^{-1} \alpha \gamma \cdot u^{-1} \in N(L, \cdot)$ for all $u \in L$ and all automorphism $\gamma$ of $(L, \cdot)$. Hence $\gamma^{-1} \alpha \gamma \in S(L)$ for all $\alpha \in S(L)$ and all automorphism $\gamma$ of $(L, \cdot)$. So $S(L)$ is indeed normal in the automorphism group of $A(L)$ of $(L,$.$) .$ 


\section{Bryant-Schneider group}

Theorem 4.1. Let $(L, \cdot)$ be a $C$-loop, an element $\theta$ of the Bryant-Schneider group of $L$ is an automorphism of $L$ provided

$$
<\theta R_{g^{-1}}, \theta L_{f^{-1}}, \theta>
$$

is an autotopism of $(L,$.$) if f$ and $g$ are elements of the nucleus of $(L,$.$) .$

Proof : Let $(L,$.$) is a C-loop then$

$$
<R_{y^{-1}} R_{y^{-1}}, L_{y} L_{y}, I>
$$

is an autotopism for all $x \in L . \theta \in B S(L,$.$) imply that <\theta R_{g^{-1}}, \theta L_{f^{-1}}, \theta>$ is also an autotopism for some $g, f \in(L,$.

Hence $<\theta R_{g^{-1}}, \theta L_{f^{-1}}, \theta><R_{y^{-1}} R_{y^{-1}}, L_{y} L_{y}, I>=$ $<\theta R_{g^{-1}} R_{y^{-1}} R_{y^{-1}}, \theta L_{f^{-1}} L_{y} L_{y}, \theta>$ is an autotopism of for all $y \in L$ and some $g, f \in L$. Since $(L,$.$) is an alternative property loop, then$

$$
R_{y^{-1}} R_{y^{-1}}=R_{\left(y^{-1}\right)^{2}}=R_{\left(y^{2}\right)^{-1}}
$$

and $L_{y} L_{y}=L_{y^{2}}$ therefore $<\theta R_{g^{-1}} R_{y^{-1}} R_{y^{-1}}, \theta L_{f^{-1}} L_{y} L_{y}, \theta>=$ $<\theta R_{g^{-1}} R_{\left(y^{2}\right)^{-1}}, \theta L_{f^{-1}} L_{y^{2}}, \theta>$. If $g=\left(y^{2}\right)^{-1}$ and $f=y^{2}$ we obtain $<$ $\theta, \theta, \theta>$ Hence $\theta$ is an automorphism of $(L,) . g=.\left(y^{2}\right)^{-1}$ and $f=y^{2}$ implies that $f=g^{-1}=y^{2}$. Then it follows that $f$ and $g$ are elements of $N(L,$.$) the nucleus of (L,$.$) since the square of every element y \in L$ belongs to $N(L,$.$) .$

Theorem 4.2. Let $(L,$.$) be a C$-loop and let $\theta \in S(L,$.$) (the symmetric$ group of $L$ ). Then $\theta \in B S(L,$.$) if there is a unique \alpha \in P(L,$.$) (the$ set pseudo-automorphisms of $(L,)$.$) and a unique f \in N(L,$.$) such that$ $\theta=\alpha R_{f}\left(\alpha=\theta R_{f}^{-1}\right)$.

\section{Proof :}

Let $(L,$.$) be a C-loop then$

$$
A=<R_{x^{-1}} R_{x^{-1}}, L_{x} L_{x}, I>
$$

an autotopism of $(L,$.$) for all x \in L$.

$B=<I, R_{x^{2}}, R_{x^{2}}>=<R_{x^{2}}, \rho R_{x^{2}} \rho, I>$ is also an autotopism for all $x \in L$. Therefore by Bruck[4]

$$
B A=<R_{x^{2}}, \rho R_{x^{2}} \rho, I><R_{x^{-1}} R_{x^{-1}}, L_{x} L_{x}, I>=<I, \rho R_{x^{2}} \rho L_{x} L_{x}, I>
$$


is an autotopism for all $x \in L . \theta \in B S(L,$.$) implies that$

$$
C=<\theta R_{f^{-1}}, \theta L_{g^{-1}}, \theta>
$$

is an autotopism for some $f, g \in L$

$$
\begin{aligned}
C B A= & \theta R_{f^{-1}}, \theta L_{g^{-1}}, \theta><I, \rho R_{x^{2}} \rho L_{x} L_{x}, I>= \\
& <\theta R_{f^{-1}}, \theta L_{g^{-1}} \rho R_{x^{2}} \rho L_{x} L_{x}, \theta>
\end{aligned}
$$

which implies that $<\alpha, \theta L_{g^{-1}} \rho R_{x^{2}} \rho L_{x} L_{x}, \alpha R_{f}>$ is autotopism of for some $f, g \in Q$ and all $x \in L$. Now if

$$
<\alpha, \theta L_{g^{-1}} \rho R_{x^{2}} \rho L_{x} L_{x}, \alpha R_{f}>
$$

is an autotopism we have $s \alpha . t \beta=\left(\right.$ s.t) $\alpha R_{f}$ for all $s, t \in L$ where $\beta=$ $\theta L_{g^{-1}} \rho R_{x^{2}} \rho L_{x} L_{x}$. If $s$ is set to be $e$ in the last autotopism and noting that $e \alpha=e \theta R_{e \theta}=e$ we get $\beta=\alpha R_{f}$ therefore $\left\langle\alpha, \alpha R_{f}, \alpha R_{f}\right\rangle$ is an autotopism of $(L,$.$) for some f \in L$ hence $\alpha$ is a pseudo-automorphism with companion $f . \theta=\alpha R_{f}$ implies that the elements of the Bryant-Schneider group of a C-loop $(L,$.$) can be expresses in terms of pseudo-automorphisms$ $P(L,$.$) and right translations of elements of the nucleus of (L,$.$) . To show$ uniqueness, let $\alpha_{1} R_{x_{1}}=\alpha_{2} R_{x_{2}}$ where $\alpha_{1}, \alpha_{2} \in P(L,$.$) and x_{1}, x_{2} \in N(L,$.$) .$ Then $\alpha_{2}^{-1} \alpha_{1}=R_{x_{2}} R_{x_{1}}^{-1}$ which implies that $e \alpha_{2}^{-1} \alpha_{1}=e R_{x_{2}} R_{x_{1}}^{-1}$. Then we observe that $e=x_{2} x_{1}^{-1}$ and therefore $x_{1}=x_{2}$. It the follows that $\alpha_{1}=\alpha_{2}$.

Remark 4.1. Robinson[21] considered the Bryant-Schneider group of a Bol loop and found out that they can be expressed as a product of pseudoautomorphisms and right translations. Theorem 2.2 above shows that the Bryant-Schneider group of a C-loop can also be expressed in the same way. This further emphasis the fact that C-loops are analogous to Moufang loops since Moufang loops satisfies the Bol identities(right and left).

Theorem 4.3. Let $(L,$.$) be a C-loop. If x, y \in Q$, let $\odot$ be a binary operation defined on the pseudo-automorphism $P S(L,$.$) by$

$$
\alpha \odot \beta=\alpha R_{x} \beta R_{y} R_{(x \beta . y)^{-1}}
$$

for all $\alpha \beta \in P S(L,$.$) . Let H=P S(L,) \times$.$Q and for$

$$
(\alpha, x) \circ(\beta, y)=(\alpha \odot \beta, x \beta . y) .
$$

Then $(H, \circ)$ a group which is isomorphic to $B S(L,$.$) .$ 


\section{Proof :}

Let $\alpha, \beta \in P S(L,$.$) and let x, y \in N(L,$.$) the nucleus of (L,$.$) . Then$ we know from the immediate preceding theorem that there exist unique $\delta \in P S(L,$.$) and unique z \in N(L,$.$) such that \alpha R_{x} \beta R_{y}=\delta R_{z}$. Thus we observe that

$$
(u \alpha . x) \beta y=u \delta . z
$$

for all $u \in L$. If we set $u=e$ we obtain $x \beta . y=z$. Therefore $\alpha R_{x} \beta R_{y}=$ $\delta R_{(x \beta . y)^{-1}}$ and so

$$
\delta=\alpha R_{x} \beta R_{y} R_{(x \beta . y)^{-1}}=\alpha \odot \beta
$$

Hence $\odot$ is a closed binary operation of $P S(L,$.$) . It is also obvious now that$ $(\alpha, x) \mapsto \alpha R_{x}$ provided $x \in N(L,$.$) gives an isomorphism of (H, \circ)$ onto the $B S(L,$.$) of a C-loop. Hence the Bryant-Schneider group of a C-loop is a$ form generalized holomorph of the loop.

Theorem 4.4. A finite C-loop is isomorphic to all its loop isotopes if

$$
[(L, .): N(L, .)]^{2}=[P S(L, .): A(L)]
$$

where $A(L)$ is the automorphism group of $(L,$.

\section{Proof :}

By Theorem 4.2 it is clear that $|B S(L,)|=.|L \| P S(L,)$.$| . By Bryant \&$ Schneider[2] $(L,$.$) is isomorphic to all its loop isotopes if$

$$
|L|^{2}|A(L, .)|=|B S(L, .)|\left|N_{\mu}(L, .)\right|
$$

But in a C-loop the nuclei coincide hence $\left|N_{\mu}(L,).\right|=|N(L,)$.$| . Now by$ Theorem $4.2|B S(L,)|=.|P S(L,)|.|N(L,)$.$| and therefore we have$

$$
|L|^{2}|A(L, .)|=|P S(L, .)||N(L, .)|^{2}
$$

which implies that

which is the same as

$$
\left[\frac{|L|}{|N(L, .)|}\right]^{2}=\frac{|P S(L, .)|}{|A(L, .)|}
$$

$$
[L: N(L, .)]^{2}=[P S(L, .): A(L, .)]
$$

as required.

Corollary 4.1. Let $(L,$.$) be a C-loop then$

$$
[P S(L, .): A(L, .)] \neq 4
$$

Proof :

The proof follows directly from Lemma 2.9 of [20] and Theorem 4.4 


\section{Acknowledgement}

The first author wishes to express his profound gratitude to the members of staff of the Faculty of Mathematical Sciences, University for Development Studies(UDS), Navrongo Campus, Ghana for hospitality. This manuscript was initially conceived and later written-up during visits to UDS as AAU fellow. .

\section{References}

[1] J. O. Adeniran, On Some Maps of Conjugacy Closed Loops., An. Ştiinţ. Univ. "AL. I. Cuza". Iasi. Mat. 50(2004), pp.267-272, (2004).

[2] Bryant, B.F. \& Schneider, H. Principal loop-isotopes of quasigroups, Can. Jour. Math., 18, pp. 120-125, (1966).

[3] R. H. Bruck, Contribution to the Theory of Loops., Trans. Amer. Math. Soc., 55, pp. 245-354, (1946).

[4] R. H. Bruck, A Survey of Binary Systems., Springer-Verlag, BerlinGöttinge-Heidelberg., (1966).

[5] O. Chein, A short note on supernuclear (central) elements of inverse property loops, Arch. Math., 33, pp. 131-132, (1979).

[6] V. O. Chiboka, The Study of Properties and Construction of Certain Finite Order G-loops., Ph. D. Thesis (1990), Obafemi Awolowo University, Ile-Ife, Nigeria., 127pp.

[7] V. O. Chiboka and A.R.T. Solarin, Holomorphs of Conjugacy Closed Loops., Scientific Annals of "AL.I.CUZA"., 38, pp. 277-283, (1991).

[8] V. O. Chiboka and A.R.T. Solarin, Autotopism Characterization of G-Loops., An. Ştiint. Univ. "AL.I.Cuza". Iasi. Mat., 39, pp. 19-26, (1993).

[9] F. Fenyves, Extra Loops I, Publ. Math. Debrecen, 15, pp. 235-238, (1968). 
[10] F. Fenyves, Extra Loops II, Publ. Math. Debrecen, 16, pp. 187-192, (1969).

[11] T. G. Jaiyeola, An Isotopic Study of C-loops., M. Sc. Dissertation (2005), University of agriculture, Abeokuta, Nigeria.

[12] M. K. Kinyon, J. D. Phillips and P. Vojtěchovský , C-loops : Extensions and construction, J. Alg. \& its Appl. (to appear).

[13] M. K. Kinyon, and K. Kunen, The Structure of Extra Loops., 6(, pp. $1-20,(2007)$.

[14] K. Kunen, Quasigroups, Loops and Associative Laws., J. Alg. 185, pp. 194-204, (1996).

[15] K. Kunen, Moufang Quasigroups., J. Alg. 183, pp. 231-234, (1996).

[16] H.O. Pflugfelder, Quasigroups and Loops: Introduction., Sigma Series in Pure Math. 7, Heldermann Verlag, Berlin, 147, (1990).

[17] H.O. Pflugfelder, Historical notes on Lopp Theory., Comment. Math. Carolinae., 4, 2, pp. 359-370, (2000).

[18] J. D. Phillips and P. Vojtěchovský, The varieties of loops of BolMoufang type, Alg. Univ., 53(3), pp. 115-137, (2005).

[19] J. D. Phillips and P. Vojtěchovský, The varieties of quasigroups of Bol-Moufang type : An equational reasoning approach J. Alg., 293, pp. 17-33, (2005).

[20] J. D. Phillips and P. Vojtěchovský, C-loops ; An Introduction, Publ. Math. Debrecen, 68(1-2), pp. 115-137, (2006).

[21] Robinson, D.A. The Bryant-Schneider group of a loop, Ann. de la Soc. Sci. de Bruxelles, 94, pp. 69-81, (1980)

[22] V.S. Ramamurthi and A.R.T. Solarin, On Finite RC-loops., Publ. Math. Debrecen., 35, pp. 261-264, (1988).

[23] D.A Robinson, Holomorphy Theory of Extra Loops., Publ. Math. Debrecen., 18, pp. 59-64, (1971).

[24] D.A Robinson, A Special Embedding of Bol-Loops in Groups., Acta Math. Hungaricae., 18, pp. 95-113, (1977). 
[25] A. R. T. Solarin, On the identities of Bol-Moufang type, Koungpook Math. J., 28(1), pp. 51-62, (1998).

\section{John Olusola Adéníran}

Department of Mathematics

University of Agriculture

Abeokuta 110101

Nigeria

e-mail : adeniranoj@unaab.edu.ng ; ekenedilichineke@yahoo.com

\section{Yakub Tunde Oyebo}

Department of Mathematics

Lagos State University

Ojo,

Nigeria

e-mail : oyeboyt@yahoo.com

and

\section{Daabo Mohammed}

Department of Mathematics

University for Development Studies

Tamale

Ghana

e-mail : daabo2005@yahoo.com 